\title{
A Simple and Uniform Way to Introduce Complimentary Asynchronous Interaction Models in an Existing Document Analysis System
}

\author{
Joseph CHAZALON and Bertrand COÜASNON \\ Université Européenne de Bretagne \\ INSA, IRISA (UMR 6074), Rennes, France \\ \{joseph.chazalon,bertrand.couasnon\} @irisa.fr
}

\author{
Aurélie LEMAITRE \\ Université Européenne de Bretagne \\ Univ. de Rennes 2, IRISA (UMR 6074), Rennes, France \\ aurelie.lemaitre@irisa.fr
}

\begin{abstract}
Extracting and indexing meaningful contents from degraded documents, like historical ones, is a challenging problem. Existing analysis systems usually rely on a manual correction of results during the post-processing stage, and cannot make use of external information to adapt their response. This paper presents how an existing document analysis system can be easily adapted to enable an efficient interaction during the analysis stage, and benefit from external information. We identify the minimal architecture required, and we detail the two complimentary interaction models we propose: a directed interaction model which allows to handle cases where errors can be automatically detected; and a spontaneous interaction model which permits to cope with the other cases. Both models are asynchronous to prevent the human operator or the system from waiting for each other during document processing. They are based on a common foundation which uses standard exception-like mechanisms to implement error detection, correction and recovery aspects. Our system was tested on several tasks. For instance, for the transcription of handwritten words in documents dating from the 18th century, where we were able to diminish the human workload by $28 \%$ for an overall recognition rate of $80 \%$.
\end{abstract}

Keywords-human interaction; asynchronous interaction; interaction models; degraded documents; historical documents

\section{INTRODUCTION}

Difficulties encountered during content extraction and indexing in old and degraded documents have at least two main causes. First, the media is damaged, and the content is altered. Documents are noisy channels which require error-correcting analysis methods. Second, both contents and structures in historical collection can vary a lot: printed and handwritten contents can be mixed, writers can change, vocabulary is outdated or unknown in advance (family names for instance), structures are not constrained by some modern formating system... Models we use tend to be unreliable as unexpected elements will very probably be encountered in the important amounts of pages we process.

These causes lead to important amounts of errors during mass processing of documents. Contextual dependencies between elements increase the final amount of manual corrections needed. It is often more efficient to let a human operator manually produce the results instead of correcting all the errors after the processing. Therefore, an analysis system should make use of external information during the processing stage to improve its response to past and future pages and globally reduce the amount of human work required. This interaction must be asynchronous to prevent the human and the system from waiting for each other.

This paper details how, using a uniform basis, complimentary interaction models can be easily implemented in an analysis system. In Sec. II, we review several existing interactive approaches for document processing and show that two interaction models are necessary. In Sec. III, we present the minimal architecture required to enable those models. In Sec. IV, we focus on how we transformed our page analyzer using a common basis for both models, easy to adapt to other systems. In Sec. V, we illustrate the capabilities of such system with two experiments we made.

\section{RELATED WORK}

Few document analysis systems allow human operators to interact with them during the analysis stage to guide or correct their response. Some systems among those few illustrate well the challenges we face.

The Edelweiss system [1] proposes a first kind of interaction. It process document pages and progressively produces an XML structure a human operator can edit at some points in the analysis process. On the one hand, it permits to influence the response of the system for the rest of the page processing by virtually transforming any element in the document structure, but on the other hand, it does not reconsider previous intermediary results, and does not validate humanprovided information against some document model.

The smartFIX system [2] relies heavily on human interaction. It is designed for mass processing of business documents, like medical invoices, and automatically triggers business transactions. It assigns a confidence score to elements it extracts and recognizes, and asks for a human validation for suspicious ones, or for a correction for problematic ones. While this approach permits an homogeneous integration of human knowledge in the process, it limits the 
modifications made by a human to final results, preventing to correct original errors and to guide the analysis.

Nagy and Veeramachaneni explore, in [3], some links between document processing and interaction: they suggest that "the operator should not even have to look at data that the system had no trouble in classifying", and also that "every interaction should be utilized by the system to improve subsequent classification". There first requirement implies a "machine-initiated interaction", where the human operator reacts to problems detected. However, the detection of errors and the localization of their cause cannot always be done automatically, while it is still necessary to replace some parts of the automated analysis by human processing.

The ability to detect errors is the key here, and we propose to distinguish interaction which is "directed" (by automatic error detection) [4], where error detection is possible, and where a question (which requires an answer) can be asked to a human operator; and interaction which is "spontaneous" [5] for cases where error detection is not possible. Spontaneous interaction is "human-initiated", as the automated system will have to react to external information, and use it to improve its response for a given page. The following sections present how an iterative analysis of pages permits to adapt the system response, and how a common foundation enables both directed and spontaneous interaction models. System improvement using machine learning will not be discussed, even if our architecture supports it.

\section{ITERATIVE ANALYSIS ARCHITECTURE FOR HUMAN INTERACTION}

To enable an asynchronous between a human operator and a document analysis system during the analysis stage, and permit to reconsider previous results for the pages processed, we need: i) an iterative analysis of pages; ii) a visual memory structure; iii) an architecture for collection processing. We now detail those aspects, and show that the page analyzer is the key component to make use of external information.

\section{A. Iterative Analysis of Pages}

The simplest iterative analysis scenario we use is the one where we first process all the pages of a collection automatically, then we let the human operator asynchronously provide necessary information about pages. Each page is then reprocessed using external information, and this scheme is repeated as many times as needed. At each iteration, new information about the page being reprocessed is provided.

To enable such approach, both the system and the human operator have to be given the same goal, and the ability to exchange information about the content of the pages, according to a previously established protocol. As interaction must be asynchronous, the system has to be able to store information associated to pages, and therefore it should be able to process them independently from the human.

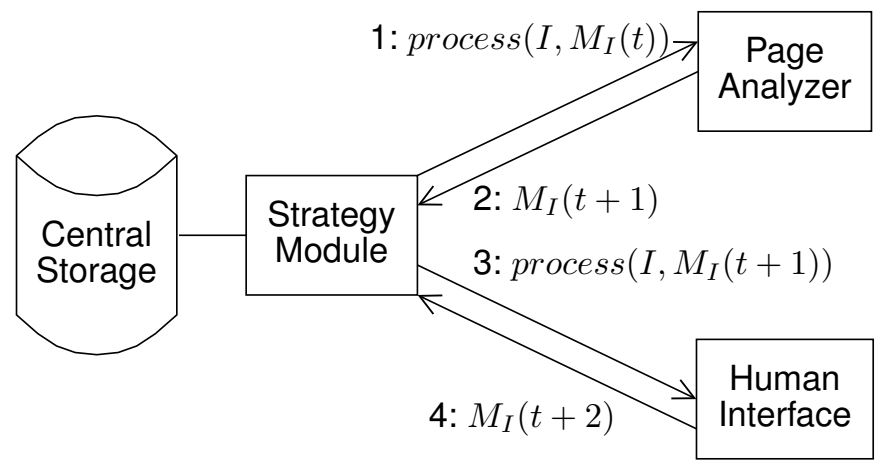

Figure 1. A single step (repeated as many times as needed during the iterative analysis) of a possible information exchange about a given image between a page analyzer and a human interface, showing the implied components of the architecture and the visual memory $M_{I}$ associated to the image $I$. At time $t$, the strategy modules first invokes the page analyzer on the image $I$, with the memory $M_{I}(t)$. The strategy module gathers $M_{I}(t+1)$ after the analysis, and transfers it to the human interface, which, in turn, produces $M_{I}(t+2)$ and sends it back.

The human operator provides as much information as required (directed model), and may be able to provide extra elements (spontaneous model). Questions of a directed interactive session could be "Where is the recipient bloc of this mail?", or "What is the value of this number (zone)?". Answers and information provided during a spontaneous interactive session are we similar, and could be "The recipient is here (zone).", "The value of this number is 27.”, or "The right place for the sum column of this bill is here (zone)."

\section{B. Visual Memory}

The visual memory is a persistent data structure associated to each page which is used for information exchange between processes, but also during document analysis in order to reintegrate external information and use it. It is an associative structure that each process can change, and which gives to each data a corresponding shape and position in its image's referential. This structure is very strongly integrated in the page analyzer, to enable the fusion between the current image content and external information during page analysis, and to guarantee that, at each moment, external data is available and that creation, deletion and modification of elements contained in the visual memory is possible.

As an example, the content associated to some image containing a table of numbers could be composed of geometric objects indicating column types, recognized numbers and their values, suspicious numbers, etc. We note $M_{I}(t)$ the visual memory related to some image $I$ at time $t$.

\section{Architecture for Collection Processing}

The iterative analysis of document pages is made possible thanks to the following components, illustrated in Fig. 1.

Strategy Module: It manages the invocation of the different processes during the analysis and their communication. This is the abstraction level where the analysis scenario 
is played, and where collection properties can be used: for instance, in [6], we grouped questions about visually similar words from various pages to reduce the number of manual work required to answer and perform the transcription.

Central Database: The central database stores the visual memories associated to each page in the whole collection. It provides a collection-centric view about the knowledge about the pages which progressively grows.

Page Analyzer: The page analyzer uses a document model to try to locate and recognize contents from a given image $I$ and its associated visual memory $M_{I}(t)$. The iterative analysis scheme allows it to transform $M_{I}(t)$ and return an updated content for $M_{I}(t+1)$ to the strategy module when done. At each iteration, it reprocesses the whole image (we integrated several optimizations to keep some results and avoid wasting too much time) and makes use of external information which was asynchronously provided. This permits to produce new structured results based on new intermediary results, all validated against the document model. In a directed interaction model, the page analyzer can generate questions indicating its lack of knowledge.

Human Interface: When solicited by the strategy module, the human interface enables a human operator to change the content of the visual memory.The page analyzer and the human interface must produce similar elements in the visual memory of each image, as they have to collaborate in such a way that the human operator may sometimes substitute himself to parts of the automated analysis to produce intermediary results.

\section{UNIFORM IMPLEMENTATION FOR DIRECTED AND SPONTANEOUS INTERACTION MODELS}

In previous papers, we separately demonstrated the interest of a directed (see [4], [6], and Sec. V-A) and of a spontaneous (see [5] and Sec. V-B) interaction models. When used appropriately, they permit to reduce the amount of manual corrections required, for a given quality level, when compared to manual correction during post-processing.

This section shows how we integrated those two interaction models in a same page analyzer, thanks to a versatile implementation of the the directed interaction model which addresses not only specific cases where automatic error detection is possible, but also supports cases where no error detection is required. We briefly describe the DMOS-P system for which the prototype implementation was designed, then we focus on each interaction model and show they can be implemented using the same foundations.

\section{A. DMOS-P Framework and Language}

DMOS-P [7] is a concept-driven grammatical document analysis method. It uses a bi-dimensional extension of Definite Clause Grammars, called Enhanced Position Formalism (EPF), to describe pages. The following example illustrates its syntax: to recognize $A$, we try, at the top of the image, to recognize $B$, and if it fails, we try, at the bottom, to recognize C. Derivation is noted ": :=", concatenation is noted " $\&$ " and alternatives are expressed with multiples derivations for a same left-hand part.

A : : = AT (top) \&\& B. $\%$ clause 1, tried 1st

$\mathrm{A}::=\mathrm{AT}$ (bottom) \&\& C. ․ tried if 1 fails

The "AT" operator is used for the positioning the analyzer's "reading head": it defines a zone in the image plan, and only elements which are present in this zone will be considered in the rule which follows this operator.

Like attribute grammars, rules have input and output parameters indicated by "+" and "-" signs, as illustrated by:

recognizenumber (+NumPos, -Value) : := callClassifier(+NumPos, -Value).

\section{B. DMOS-P Extensions for Directed Interaction}

The directed interaction model we propose was introduced in [4] and its efficiency was demonstrated in [6]. This model relies on the introduction of 3 new operators in the page description language. The semantics associated to those operators permit to express properties about pages. Their implementation allow to change the behavior of the page analyzer to automatically handle the interaction with a human operator or other components. It is based on an asynchronous information exchange made of questions and answers and using the visual memory, throughout the various page analyzer invocations at each analysis iteration.

The visual memory carries (and locates in the image referential) questions and answers using the containers:

$Q($ Text, DataType $)$

A(Data)

Text gives information about the problem and DataType indicates the expected type for the content of the answer, which is stored in its Data attribute.

From a technical point of view, the operators were implemented using a continuation mechanism in the logical programming language used in DMOS-P. Continuations are, in this case, constructs very similar to the standard exception mechanism commonly used in imperative languages: our approach should be easily adapted to other systems.

Our implementation enables an asynchronous error detection and correction, and also allows to recover from errors during page analysis. In order to make a clear analogy between our new operators and exception handling, we chose names reflecting the behavior they produce during the analysis. Those operators are designed to: i) detect errors and ask questions; ii) ensure that answers are used by the analyzer to make progress instead of asking the same questions forever; and iii) make as much progress as possible in independent parts of the analysis if a problem arises.

1) Asking Questions (Error Detection): This operator is used to indicate that some part of the page cannot be 
understood properly. It can be to indicate that no valid interpretation could be proposed (all alternatives failed); or that recognized element are inconsistent with each other (non incrementing page numbers for example); or simply that the current case should better be processed by a human (not implemented, not reliable, etc.). The syntax and the algorithm describing the behavior of the page analyzer are:

raisequestion (+Text, +Zone, +DataType)

When called, it 1) adds a question in the visual memory $M_{I}$, with shape and position defined by +Zone to locate the issue; and 2) continues the analysis just after the latest invocation of catchquestion. The following example illustrates how to ask a question if, in some mail we try to recognize, we cannot find the recipient.

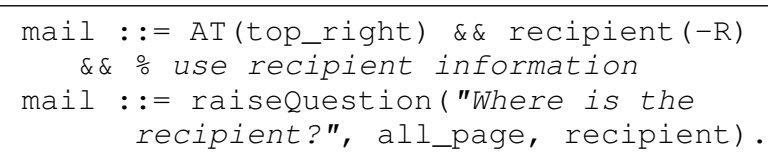

Answering the questions is done outside of the page analyzer, in the human-machine interface, after the automatic step of the current iteration has been completed. Answers are stored in the visual memory and will be usable in the next iteration.

2) Using Answer (Error Correction): This operator is used to indicate the part of the document model which need to be ignored when an appropriate answer exists in the visual memory. This part of the page model is supposed to produce a result, unless an error occurs and then the automated analysis will be replaced by a human operator, or another automated process. This operator is the one which enables both interaction models, as we will see in Sec. IV-C. Syntax and implementation algorithm are:

getAnswerOrTry (+DataType, -Result, +Rule)

where +DataType indicates the type of the content of acceptable answers, and $+R u l e$ is a rule which has a unique output parameter whose type is also +DataType. The type of the element contained in the answer is the same as the type of the result produced by the automatic rule.

When called, it looks at the current search position for an answer $A($ Data $)$ in $M_{I}$ where the type of Data is +DataType. If it exists, the value of -Result is Data. Otherwise, it invokes the rule + Rule and the value of the output parameter of + Rule is used as value for -Result.

In order to avoid asking questions indefinitely, we need to ensure that if some appropriate answer was provided, the original question will not be asked again. To do so, we control that usage of raiseQuestion is only made inside rules annotated with a getAnswerorTry with the same DataType. The following example illustrates this usage.

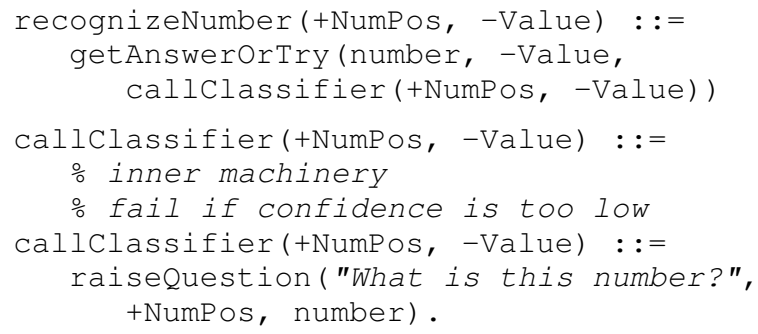

3) Continuing the Analysis (Error Recovery): This operator is used to indicate the scope of some error in the page model. Outside this scope, the analysis is not impacted by such error and can be continued if it occurs. This operator is noted:

catchQuestion (+Rule)

where +Rule is a rule which may have any parameter. When called, it invokes the rule +Rule and catches any interaction request raised with raiseQuestion in that rule. If no question is raised, the output parameters of +Rule are well defined, otherwise they are left uninstantiated. If, for some table recognition task we can process rows independently, then we could write:

rowlist $::=$ catchQuestion(one_row) \&\&

\% detect other rows independently

\section{Introducing Spontaneous Interaction}

Spontaneous interaction relies on a simpler protocol than directed interaction. There is no need to detect errors and ask questions, and no need to recover from error. However, the ability to replace some parts of the automated analysis by a manual edition made by a human operator is necessary. Therefore, the getAnswerorTry operator contains all the logic we need to enable spontaneous interaction: if we allow (thanks to some modification in the protocol defined in the strategy module) the human operator to provide information with the right type, located at the right place in the image referential, then the analyzer will automatically use it and will skip specific parts of the automated analysis process. As a result, we should not call "answers" every piece of information provided by a human operator, but we should simply say "information" about image content.

This approach allows to annotate specific parts of the document model which may be manually corrected by human operators during interaction steps. This allows to correct erroneous element, or add missing results in an intermediary structure, like in the following example:

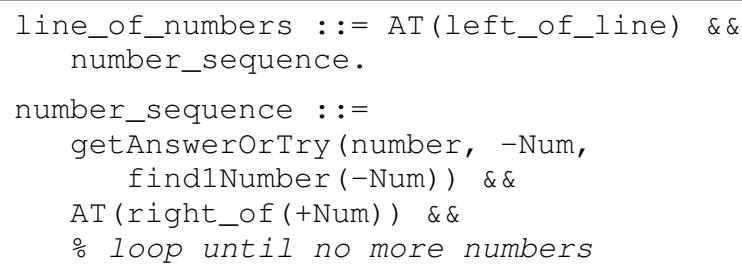


If the system misses a number, a human operator can add it in the visual memory and during the next analysis iteration, it will be used just like if it was detected in the image.

\section{Implementation Advantages}

The implementation we propose not only permits to introduce the two interaction model we propose in the same page analyzer, it also has two main advantages. The first advantage is to propose a clear separation between the page description and the implementation of the automated management of the interaction with some human operator (or other software components). It permits to keep the page description simple, and also to improve the interaction management without changing the page model. The second advantage is to integrate external information (humanprovided, system-provided, or both), exactly as if they were extracted from the image. As a result, we can assign a confidence score to external information, validate it against the document model, and use it to produce final results.

\section{Applications and Results}

\section{A. Directed Interaction for the Transcription of Documents from the $18^{\text {th }}$ Century}

The directed interaction model was used to efficiently transcript handwritten words from sales registers of the $18^{\text {th }}$ century [6]. In the tests we made, 70 documents (1206 words) were processed, and extracted words were grouped by visual similarity in clusters (making use of redundancies of several words among pages), which were manually annotated if necessary. The iterative analysis permitted to reintegrate and validate external information (extracted using collection context) within a unique page model. This global approach diminished the human labeling of words from $21 \%$ (manual labeling of all suspicious elements) to $15 \%(-28 \%)$ for an overall recognition rate of $80 \%$.

\section{B. Spontaneous Interaction to Correct Number Segmenta- tion Issues in Documents from the $18^{\text {th }}$ Century}

The spontaneous interaction model was used to correct segmentation issues during the localization of numbers in an excerpt (50 pages with 1637 numbers) of a document dating from the $18^{\text {th }}$ century [5]. Those numbers were separated by some special characters which where not always properly detected, and caused under-segmentation issues. In the tests we made, we allowed the human operator to review localization results, and to indicate the position of separators which were not detected. Using this approach, we were able to recover $40.6 \%$ of the missing numbers and to eliminate $30.0 \%$ of the erroneous zones. Correcting errors during the analysis stage allowed us to reduce by $29.8 \%$ the number of manual actions required to reach this quality level, when compared to a manual post-processing.

\section{CONCLUSION}

Interacting with a human operator during the analysis stage is necessary to process old and degraded documents. While a directed interaction model may be the most efficient approach, as asking missing information enables to optimize human interaction, the error detection this model relies on is not always possible. Spontaneous interaction is a valuable alternative in those cases, as it also permits to reduce human workload when compared to post-processing. This paper show how we introduced both directed and spontaneous interaction models in the same page analyzer to be able to benefit from both approaches. The implementation we used enables an asynchronous error detection, correction and recovery throughout the iterations of the analysis. It is based on standard programming constructs very close to exceptions and should be easily adapted to other existing systems. Doing so, making use of external information during the analysis is very easy, and we were able to benefit from human-provided information, but also information extracted from collection context: redundancies of words among pages helped us to transcript documents from the $18^{\text {th }}$ century, with fewer manual actions than before.

\section{ACKNOWLEDGMENT}

This work has been done in cooperation with the Archives départementales des Yvelines in France, with the support of the Conseil Général des Yvelines.

\section{REFERENCES}

[1] N. Roussel, O. Hitz, and R. Ingold, "Web-based cooperative document understanding," in Proc. of ICDAR, 2001, pp. 368373.

[2] B. Klein, A. Dengel, and A. Fordan, "smartFIX: An adaptive system for document analysis and understanding," in Reading and Learning, Dengel, Junker, and Weisbecker, Eds. Springer, 2004, vol. 2956 of LNCS.

[3] G. Nagy and S. Veeramachaneni, "Adaptive and interactive approaches to document analysis," in Machine Learning in Document Analysis and Recognition, ser. Studies in Computational Intelligence, S. Marinai and H. Fujisawa, Eds. Springer, 2008, vol. 90, pp. 221-257.

[4] J. Chazalon, B. Coüasnon, and A. Lemaitre, "Iterative Analysis of Pages in Document Collections for Efficient User Interaction," in Proc. of ICDAR, 2011.

[5] J. Chazalon and B. Coüasnon, "Iterative analysis of document collections enables efficient human-initiated interaction." in Document Recognition and Retrieval XIX, Proc. SPIE, 2012.

[6] L. Guichard, J. Chazalon, and B. Coüasnon, "Exploiting Collection Level for Improving Assisted Handwritten Words Transcription of Historical Documents," in Proc. of ICDAR, 2011.

[7] B. Coüasnon, "Dealing with noise in DMOS, a generic method for structured document recognition: An example on a complete grammar," in Graphics Recognition, Lladós and Kwon, Eds. Springer, 2004, vol. 3088 of LNCS. 